\title{
Targeted Therapy for Early and Locally Advanced Breast Cancer
}

\author{
Michael Untch \\ Klinik für Gynäkologie und Geburtshilfe, Helios Klinikum Berlin Buch, Germany
}

\section{Key Words}

Breast cancer: early, locally advanced .

Targeted therapy · HER2 - Neoadjuvant therapy ·

Trastuzumab - Lapatinib - Bevacizumab - Neratinib ·

T-DM1 $\cdot$ Pertuzumab

\section{Summary}

The treatment of patients with breast cancer continues to evolve, with cytotoxic chemotherapy, endocrine therapy, and molecular targeted therapies representing the backbones of modern systemic breast cancer treatment. As we learn better to understand the biology of breast cancer cells, therapies to target specific pathways continue to be developed with the goal of expanding available effective therapy in specific populations. Several targeted drugs with different molecular pathways have achieved approval for metastatic breast cancer, but for early breast cancer trastuzumab is the only one that is currently approved in combination with chemotherapy for adjuvant or neoadjuvant treatment in women with HER2-positive breast cancer. Lapatinib and bevacizumab are both approved for the treatment of metastatic breast cancer and are now investigated in phase III clinical trials testing their effectiveness in the treatment of early breast cancer. In this publication, we review the current status in the treatment of early and locally advanced breast cancer with molecular targeted therapies that are currently approved or in advanced clinical development.

\author{
Schlüsselwörter \\ Mammakarzinom: frühes, lokal fortgeschrittenes . \\ Zielgerichtete Therapie - HER2 - Neoadjuvante Therapie · \\ Trastuzumab - Lapatinib - Bevacizumab - Neratinib . \\ T-DM1 $\cdot$ Pertuzumab
}

\section{Zusammenfassung}

In den vergangenen Jahren hat sich die Brustkrebstherapie rasant weiterentwickelt. Neben zytotoxischen und endokrinen Behandlungsoptionen bilden zielgerichtete Therapien mittlerweile das Rückgrat moderner, systemischer Therapieansätze. Insbesondere die erweiterten Kenntnisse der Biologie der Tumorzellen haben dazu beigetragen, dass zielgerichtete Behandlungen entwickelt wurden, die das Spektrum herkömmlicher Therapien erweitern und zudem direkt auf spezielle Patientinnengruppen zugeschnitten sind. Im metastasierten Stadium stehen bereits einige zielgerichtete Therapien, die verschiedene molekulare Zielstrukturen beeinflussen, zur Verfügung. In der adjuvanten bzw. neoadjuvanten Situation ist Trastuzumab in Kombination mit Chemotherapie bei Frauen mit HER-2-positivem Mammakarzinom zugelassen. Lapatinib und Bevacizumab, die beide schon für die Behandlung von Patientinnen mit metastasierter Erkrankung zugelassen sind, werden momentan in Studien in den frühen Stadien überprüft. Zudem bieten sich weitere molekulare, zielgerichtete Therapien für die adjuvante und neoadjuvante Situation an und werden zum Teil schon in Studien getestet. Dieser Artikel möchte einen umfassenden Studienüberblick geben, inwieweit zielgerichtete Therapien in der adjuvanten und neoadjuvanten Situation bereits zum Einsatz kommen und welche Entwicklungen in nächster Zeit hinsichtlich dieser molekularen Ansätze zu erwarten sind.

\begin{tabular}{ll}
\hline KARGER & @ 2010 S. Karger GmbH, Freiburg \\
Fax +497614520714 & $\begin{array}{l}\text { Accessible online at: } \\
\text { www.karger.com/brc } \\
\text { Information@Karger.de } \\
\text { www.karger.com }\end{array}$
\end{tabular}




\section{Introduction}

The topic of selective and targeted therapy for breast cancer is not new, though we think of targeting growth signals as a recent development. Already in 1896, Beatson [1] introduced ovariectomy into clinical practice. With the discovery of the estrogen receptor (ER) and with our evolving understanding of the biology of the ER pathway, the development of targeted agents to modulate the activity of this pathway (selective estrogen receptor modulators, SERMS), to inhibit the production of the ligand (aromatase inhibitors), and to downregulate receptor expression and activity (fulvestrant) has markedly improved outcomes of localized and advanced breast cancer expressing the ER [2]. While hormonal therapy is not traditionally defined as targeted therapy, the ER receptor remains the most important growth factor receptor for breast cancer cells, and adjuvant hormonal therapies have a higher impact on breast cancer recurrence and survival than any other treatment. Today, targeted drugs interfere with specific molecules that are needed for carcinogenesis, tumor growth, and metastasis. The humanized monoclonal antibody trastuzumab was developed as a therapy targeted against the human epidermal growth factor receptor 2 (HER2) which is overexpressed in roughly one fourth of patients with invasive breast cancer. Readily available markers of overexpression and/or gene amplification of HER2 in tumor tissue predict the activity of this agent, and exclude those who will not benefit from this therapy. Randomized trials have demonstrated a survival benefit associated with the introduction of this agent in addition to chemotherapy in all stages of disease in women with breast cancers that overexpress the HER2 protein or show amplification of the HER2 gene [3-8]. As the understanding of the biology of breast cancer evolves, several other important intracellular pathways have been identified as targets for novel therapeutic agents, including other members of the HER family, pro-angiogenic pathways, proliferative pathways, pathways of cell cycle regulation, apoptosis pathways, and many others (fig. 1). It is also becoming clear that the complex interaction of these pathways will likely require multiple targets to be inhibited to optimize cytotoxicity and overcome resistance mechanisms. For early and potentially curable breast cancer, today's only approved targeted drug is trastuzumab, but there are several new drugs that are investigated in trials. In the following chapters we review the current status of recommendation and the future aspects of targeted therapy in early and locally advanced breast cancer.

\section{Targeted Therapy in HER2-Positive Early Breast Cancer}

\section{Trastuzumab}

Five large randomized trials including more than 13,000 patients have defined the role of trastuzumab in adjuvant ther-

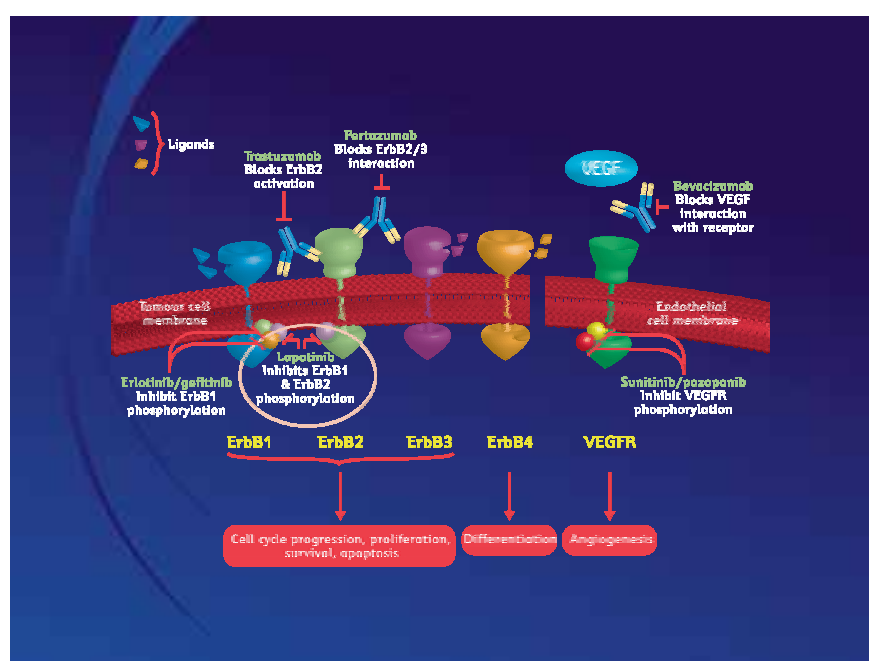

Fig. 1. Pathways in breast cancer and targets for therapeutic agents.

apy of early HER2-positive breast cancer [5-8]. These studies, together with a metaanalysis of the data [9], confirm the overall benefit of adjuvant trastuzumab therapy in HER2positive patients. Table 1 gives an overview of the published data. Pooled data of the metaanalysis show a significant reduction in mortality $(p<0.00001)$, recurrence rate $(p<0.00001)$, rate of metastasis $(\mathrm{p}<0.00001)$, and rate of secondary malignancies $(\mathrm{p}=0.007)$ with adjuvant trastuzumab therapy. The incidence of grade III/IV cardiotoxicity was higher in patients who received trastuzumab $(203 / 4,555=4.5 \%)$ than in patients without trastuzumab treatment $(86 / 4,562=1.8 \%)$. The relative likelihood of cardiotoxicity was 2.45 -fold higher in the trastuzumab groups (95\% confidence interval (CI) 1.89-3.16). Overall, symptomatic cardiac events were rare $(2-4 \%)$. The Breast Cancer Guideline of the German Gynecological Oncology Working Group (AGO) in its 2010 version recommends routine cardiac monitoring prior to and during adjuvant therapy with trastuzumab (Oxford level of evidence (LoE) 5, grade (GR) D, AGO ++; table 2). Trastuzumab can be administered sequentially, following an adjuvant chemotherapy containing anthracyclines. For taxane-containing schedules, trastuzumab may be administered concomitantly or sequentially. Treatment with trastuzumab may be given in parallel to radiotherapy. The duration of antibody treatment is 1 year.

\section{Open Questions for Adjuvant Trastuzumab}

Based on these results, the standard of care has changed in patients with HER2-positive breast cancer. But there are still some open questions that are consecutively answered by longer follow-up of the studies and additional results from new trials.

\section{Duration of Treatment}

In the majority of studies, the duration of treatment with trastuzumab has been 1 year, a condition which has been the 
Table 2. AGO Recommendations for the adjuvant therapy with trastuzumab $1 / 2010$

\begin{tabular}{|c|c|c|c|}
\hline & $\begin{array}{l}\text { Oxford } \\
\text { LoE }\end{array}$ & Grade & AGO \\
\hline Node-positive disease & $2 b$ & $\mathrm{~B}$ & ++ \\
\hline $\begin{array}{l}\text { Node-negative disease with additional } \\
\quad \text { risk factors }(\text { e.g. } \geq 1 \mathrm{~cm})\end{array}$ & $2 b$ & B & ++ \\
\hline $\begin{array}{l}\text { Node-negative disease with tumors } \\
\geq 5 \mathrm{~cm} \text { and additional risk factors } \\
\text { (if chemotherapy applicable) }\end{array}$ & 4 & C & + \\
\hline
\end{tabular}

LoE $=$ Level of evidence

rationale for the currently accepted therapeutic recommendations. The issue of shortening the duration of antibody administration to 9 weeks was highlighted in the FinHer study. It covered 1,010 patients with node-positive, high-risk breast cancer, who were randomized to receive 3 cycles of docetaxel or vinorelbine, followed by 3 cycles of fluorouracil, epirubicin, and cyclophosphamide (FEC). After completing chemotherapy, 232 women with HER2-positive breast cancer were randomized to receive trastuzumab or no further treatment. Data from a 62-months median follow-up period are available and have been presented in St. Gallen in 2009: After adjustment for lymph node status, a comparison of treatment and no treatment with trastuzumab did not reveal a significant improvement with trastuzumab. After 5 years, 83.3\% of the patients in the trastuzumab group were free of recurrence, compared to $73.0 \%$ in the control group (hazard ratio (HR) $0.65 ; 95 \%$ CI $0.38-1.12 ; p=0.12$ ) [10]. In contrast to the initial, positive results of the FinHer study, the considerably longer follow-up period did not show a significant difference. The low number of patients is regarded to be the major problem of this study. The standard duration of therapy therefore continues to be 1 year. The reduced duration of trastuzumab administration is reassessed in 2 ongoing studies, with the endpoint disease-free survival (DFS). The study NCT 00381901 by the National Cancer Institute of France [11] investigates 6 vs. 12 months of trastuzumab in node-positive and node-negative patients with operable tumors $\geq 1 \mathrm{~cm}$. The Italian Short-HER study [12] is a randomized, multicenter, phase III study with 2,500 patients being randomized to receive 4 cycles of an anthracycline-based chemotherapy, followed by 4 cycles of a taxane combined with trastuzumab, and subsequently 14 further doses of trastuzumab every 3 weeks, or three 3-week cycles of docetaxel combined with 9 doses of trastuzumab given weekly, and followed by 3 cycles of FEC.

The longer duration of trastuzumab treatment has been investigated in the HERA study. In this randomized, international, phase III study, trastuzumab was administered to 5,102 women with HER2-positive breast cancer for a period of 1 or 2 years, and both groups were compared to a chemotherapy 
Table 3. Prognosis of patients with tumors $<1 \mathrm{~cm}$

\begin{tabular}{llllll}
\hline Study [ref.] & Patients, $\mathrm{n}$ & Tumor size & Endpoint & HER2+ vs. HER2-, \% & p value \\
\hline Joensuu et al. 2003 [16] & 239 & T1a,b & 9-year DDFS & 67 vs. 95 & 0.003 \\
Rakkhit et al. 2008 [15] & 965 & T1a,b & 5-year RFS & 77.1 vs. 93.7 & $<0.0001$ \\
Amar et al. 2007 [39] & 401 & T1a,b & RFS & 92.6 vs. 98.7 & 0.007 \\
Tovey et al. 2008 [40] & 362 & T1a,b,c & 5-year BCSS & 68 vs. 96 & $<0.001$ \\
Pagani et al. 2008 [41] & 340 & T1a,b & RFS & 87.1 vs. 96.8 & NR \\
Norris et al. 2006 [42] & 326 & T1a,b,c & 10-year RFS & 75.6 vs. 82.4 & 0.66 \\
Chia et al. 2008 [17] & 225 & T1b & 10-year RFS & 68.4 vs. 81.8 & 0.312 \\
Park et al. 2009 [36] & 466 & T1a,b & 10-year DRFS/OS & $8.8 / 5.0$ & $0.003 / 0.067$ \\
\hline
\end{tabular}

BCSS = Breast cancer-specific survival; DDFS = distant disease-free survival; DRFS = distant relapse-free survival estimated, 61-month follow-up; RFS = relapse-free survival; NR = not reported. only group. Following surgery with or without radiotherapy, the participants had undergone a (neo)adjuvant treatment according to the standard procedure. Considering the significant benefit for trastuzumab in terms of DFS and overall survival (OS) shown in 2005, the patients from the observation only group were offered a switch to one of the trastuzumab groups. Of the 1,698 patients in the observation only group, 885 switched to trastuzumab. However, more than 400 patients had experienced disease progression at this time. At the Breast Cancer Conference in St. Gallen in 2009, the results from a 4-year follow-up period were presented [13]. Despite the high percentage of patients with a delayed start of the trastuzumab treatment, significantly more patients in this group had remained disease-free 4 years after randomization (76.8 vs. $72.2 \%, \mathrm{HR}=0.76$; 95\% CI $0.66-0.87$; $<<0.0001$ ), compared to the observation only group. Data to compare the 1-year and the 2-year treatment period are expected to be available in 2011.

\section{Effectiveness of Trastuzumab in Different Subgroups}

A subgroup analysis within the HERA study should provide information on the magnitude of clinical benefit in subgroups of patients defined along their node and hormonal receptor status [14]. Overall, this evaluation covered 1,703 women treated with trastuzumab for 1 year, and 1,698 women who had been monitored only. The median follow-up time was 23.5 months. Primary endpoint was DFS. The overall HR for trastuzumab compared to observation only was 0.64 (95\% CI $0.54-0.76 ; \mathrm{p}<0.0001)$. Estimated improvement of the 3-year DFS rate was between 11.3 and $0.6 \%$ in the subgroups. Best prognosis and maximum benefit, as seen in node-negative patients with tumors between 1.1 and $2.0 \mathrm{~cm}$, was similar to that of the entire cohort (HR 0.53 ; 95\% CI $0.26-1.07$; improvement of 3-year DFS rate $+4.6 \%$; $95 \%$ CI $-4.0-13.2 \%$ ). Adjuvant trastuzumab is capable of reducing the recurrence risk in all subgroups as defined by nodal and hormonal receptor status to a similarly high degree. This also applies to the subgroups of patients with small tumors. The benefit independ- ent of tumor size was pointed out in a current retrospective study [15] which showed a 5-year recurrence rate of $23 \%$ in HER2-positive breast cancer, but only $6.3 \%$ in patients with HER2-negative tumors.

\section{Adjuvant Trastuzumab Therapy in Tumors $<1 \mathrm{~cm}$}

Small node-negative breast cancers with $<1 \mathrm{~cm}$ tumor size have a favorable prognosis. Several retrospective studies have analysed the risk of recurrence among patients with tumors $\leq 1 \mathrm{~cm}$ as a function of ER and HER2 status (table 3). Registry data from Finland [16] and British Columbia [17] revealed that in T1abN0 breast cancers, HER2 overexpression was a clear risk factor for recurrence. Recurrence rates of $15-30 \%$ were detected in cancers of $1-10 \mathrm{~mm}$ in size. These risks were substantially higher than seen among similarly sized HER2negative tumors. The reports in December 2009 in the Journal of Clinical Oncology from Curigliano et al. [18] from the Istituto Europeo di Oncologia in Milan and Gonzalez-Angulo et al. [19] from the M. D. Anderson Cancer Center found much higher rates of recurrence among T1abN0 HER2-positive tumors compared with HER2-negative tumors, irrespective of ER status. Hazard estimates suggest that HER2 expression increased the risk of recurrence by two- to five-fold, with absolute risks in approximately 5 years of follow-up of 10-23\%, despite the small size of these cancers and despite the various adjuvant therapies that these patients have received. These risks are clearly lower than in other cohorts of larger and/or node-positive, HER2-positive cancers but are substantially higher than seen among small, node-negative, HER2-negative cancers, especially ER-positive, HER2-negative tumors. There is no direct evidence that trastuzumab will decrease the recurrence rate among patients with small, HER2-positive tumors, but there is considerable indirect evidence. To date, retrospective studies from the pivotal trials of adjuvant trastuzumab have not demonstrated different rates of risk reduction among HER2-overexpressing breast cancers as a function of nodal status or tumor size. This suggests that small HER2positive breast cancers $\leq 1 \mathrm{~cm}$ carry sufficient risk to consider adjuvant trastuzumab therapy which has shown to reduce the relative risk of disease recurrence by at least $50 \%$ in other 
HER2-positive patient groups. However, an accompanying editorial in the same Journal of Clinical Oncology stated that this therapy should generally be limited to patients with tumors of at least $0.5 \mathrm{~cm}$ in diameter (stage T1b or higher), since smaller size is associated with lower risk - even within the 1-cm-and-under tumor category. Trastuzumab has only been directly tested in larger, higher-risk breast cancers and is therefore recommended in consensus guidelines only for patients with tumors larger than $1.0 \mathrm{~cm}$. For tumors $0.6-1.0 \mathrm{~cm}$, the guidelines suggest trastuzumab as an option. But for those under $0.5 \mathrm{~cm}$, guidelines call for no additional therapy after surgery and radiation. Based on these data, the German AGO group concluded in 2010 that adjuvant treatment with trastuzumab might be considered for individual HER2-positive patients with tumors smaller than $1 \mathrm{~cm}$ and additional risk factors, if chemotherapy is indicated, (LoE 4; GR C, AGO+). In women with small, node-negative, HER2-positive breast tumors, shorter trastuzumab-based chemotherapy regimens that may have less short-term toxicity should be investigated. In the study NCT00542451, 400 women with stage I breast cancers are treated prospectively with paclitaxel plus trastuzumab for 12 weeks, followed by maintenance trastuzumab. We hope that this study and others will show improved tolerability and a low risk of recurrence for these patients with small tumors.

\section{Anthracycline-Free Adjuvant Treatment?}

Another issue under discussion is the importance of anthracyclines in the framework of a trastuzumab-containing adjuvant therapy. Particularly in patients with cardiovascular risks, the use of anthracyclines should be considered carefully. Data have been provided by the BCIRG-006 study which compared 2 anthracycline-containing regimens and 1 anthracycline-free regimen in 3,222 women with HER2-positive and node-positive or node-negative high-risk breast cancer [20]. Patients in a control group received a standard chemotherapy comprising 4 cycles of doxorubicin/cyclophosphamide (AC) and subsequently 4 cycles of docetaxel (AC-T). In one of the two experimental groups, participants were given trastuzumab concomitantly with docetaxel, and continued subsequently for a total of 1 year (AC-TH). In the third group, an anthracycline-free therapy comprising 6 cycles of docetaxel/ carboplatin plus trastuzumab starting with the first cycle $(\mathrm{TCH})$, was evaluated. The third interim analysis confirmed the significant benefit provided by additional trastuzumab treatment [21]. At 5 years, there were 656 events among the patients: 257 in the control group, 185 in the AC-TH group, and 214 in the TCH group. DFS was $84 \%$ for AC-TH, $81 \%$ for $\mathrm{TCH}$, and $75 \%$ for AC-T. For the primary end point of DFS, both trastuzumab-containing groups were statistically superior to the control group. Compared with the control group, the AC-TH group had a HR of 0.64 (95\% CI 0.53-0.78; $\mathrm{p}<0.001)$, and the TCH group had a HR of $0.75(95 \% \mathrm{CI}$ $0.63-0.90 ; \mathrm{p}=0.04)$. The non-anthracycline TCH regimen had a different toxicity profile than AC-TH. Some non-hematologic toxicities occurred less frequently with $\mathrm{TCH}$, including grade 3 and 4 arthralgia, myalgia, hand-foot syndrome, stomatitis, vomiting, neutropenia, and leukopenia. The lower cardiotoxicity was regarded a major advantage of the $\mathrm{TCH}$ regimen. There were differences in grade $3 / 4$ cardiac left ventricular ejection fraction (LVEF) and congestive heart failure: The AC-TH group had 21 such events, whereas the TCH group had 4 events. A full paper of the data is still pending. In May 2008, the FDA approved the anthracycline-free regimen TCH which had been evaluated in the BCIRG study. The European Authority EMEA, in contrast, declined the approval in 2008. AGO classifies TCH in 2010 as LoE 1b, Gr B, AGO +. $\mathrm{TCH}$ is an effective and safe treatment option which we would recommend to patients who are not suited to receive an anthracycline-containing adjuvant treatment. Anthracycline-free adjuvant strategies should be further investigated in clinical trials.

\section{New Approaches Targeting HER2}

Though trastuzumab-based treatments did actually improve systemic control as well as survival rates in patients with HER2-positive breast cancer, disease progression may continue in some patients due to trastuzumab resistance which can be de novo or acquired. This phenomenon arouses interest in a combination of trastuzumab and lapatinib, because either agent blocks the HER2 signaling pathway via a different mechanism of action. Furthermore, trastuzumab acts upon the extracellular domain, and lapatinib on the intracellular domain. Consequently, a combination might result in a more comprehensive inhibition of the HER2 signaling pathway and overcome potential mechanisms of resistance. Preclinical data suggested a wide lack of cross resistance of trastuzumab and lapatinib, an advantage which could be verified in clinical research. The AGO 2010 recommendations for the use of lapatinib in metastatic breast cancer are shown in table 4 .

\section{Role of Adjuvant Lapatinib in HER2-Positive EBC}

Data from a phase III study with lapatinib and capecitabine were used to create the rationale for testing this substance for adjuvant treatment [22]. The future role of lapatinib in adjuvant therapy of patients with HER2-positive tumors requires further elucidation. Throughout the world, 8,000 patients with surgically treated, HER2-positive breast cancer were in the framework of the ALTTO study which was conducted together with the Breast International Group (BIG). The majority of patients included in this 4-armed study were treated with chemotherapy containing anthracycline, which may have 
Table 4. AGO recommendations 2010 for lapatinib in metastatic breast cancer

\begin{tabular}{llll}
\hline & $\begin{array}{l}\text { Oxford } \\
\text { LoE }\end{array}$ & Grade & AGO \\
\hline $\begin{array}{l}\text { In combination with } \\
\text { Paclitaxel as 1st line }\end{array}$ & $2 \mathrm{~b}$ & $\mathrm{~B}$ & $+/-$ \\
Capecitabine as $\geq 2 \mathrm{nd}$ line & $1 \mathrm{~b}$ & $\mathrm{~B}$ & + \\
AI with ER+ disease & $2 \mathrm{~b}$ & $\mathrm{~B}$ & $+/-$ \\
Trastuzumab in heavily & $2 \mathrm{~b}$ & $\mathrm{~B}$ & + \\
pretreated patients & & & $+/-$ \\
$\begin{array}{l}\text { In patients with brain metastases } \\
\text { (radioresistant) combined with }\end{array}$ & $2 \mathrm{~b}$ & $\mathrm{~B}$ & + \\
capecitabine & & & \\
\hline
\end{tabular}

$\mathrm{LoE}=$ Level of evidence; $\mathrm{AI}=$ aromatase inhibitor; $\mathrm{ER}+=$ estrogen receptor-positive.

been followed by weekly paclitaxel. They were randomized to receive lapatinib, trastuzumab, a combination of both, or both agents sequentially. The primary endpoint was DFS. The TEACH study (Study of Tyverb Evaluation After Chemotherapy) investigated the safety and efficacy of lapatinib in the treatment of patients with early stage breast cancer overexpressing ErbB2 (HER2) after adjuvant therapy without trastuzumab. DFS time with lapatinib compared to placebo was the primary study endpoint. The recruitment phase for this randomized, placebo-controlled, phase III study was completed with 3,164 included patients, the data are currently evaluated.

\section{Targeted Therapy in HER2-Negative Breast Cancer}

Bevacizumab showed prolonged time to progression in metastatic disease (ECOG study, AVADO study, RIBBON 2.3). Ongoing adjuvant trials (1 phase II study, and 3 phase III studies E5103, BEATRICE, and BETH), evaluate efficacy and tolerability of bevacizumab combined with standard chemotherapies. Potentially life-threatening side effects of the antiangiogenic treatments will be particularly in focus. These include hypertension, proteinuria, thromboembolic events, bleeding, and gastrointestinal perforations. Heart failure will be monitored only in patients with simultaneous or preceding anthracycline treatment. Currently, no data on toxicity and efficacy are available, but first data are expected in 2011.

\section{Clinical Results - Neoadjuvant Therapy}

One aim of the treatment of locally advanced disease, particularly of inoperable disease, is the reduction of tumor burden. To maximize the chance of converting unresectable breast cancer into resectable breast cancer, a regimen with the high- est likelihood of shrinking the tumor is desired. Numerous large, randomized trials could show that there is no difference in long-term survival between adjuvant and neoadjuvant therapy. The NSABP B-18 study has been pivotal for the equiefficacy of the neoadjuvant treatment. In this prospective randomized trial, 1,523 patients with inoperable breast cancer were recruited to receive 4 cycles of AC $\left(60 / 600 \mathrm{mg} / \mathrm{m}^{2}\right)$ prior to or after surgery. After a median follow-up of 9 months, the data revealed identical OS (70 vs. $69 \%$ ) and DFS (55 vs. $53 \%$ ) [23]. The percentage of breast conserving surgery was increased by preoperative chemotherapy from 60 to $67 \%$. Patients with large tumors $(>5.1 \mathrm{~cm})$ benefitted the most [24]. Clinical as well as histopathological response correlated significantly with the individual prognosis. With a pathologic complete remission (pCR) obtained, the likelihood of dying from breast cancer was relatively reduced by $50 \%$, as compared to the entire study population. It was shown that the ratio of pCR of HER2-positive disease could be improved considerably, and exceeded that of HER2-negative tumors. One of the first clinical trials to confirm this approach was the AGO study named TECHNO (Taxol-Epirubicin-Cyclophosphamide-Herceptin Neoadjuvant) [25]. All 230 patients included into this multicenter phase II study had HER2-positive, locally advanced breast cancer and received 4 cycles of epirubicin and cyclophosphamide (EC), followed by 4 cycles of paclitaxel every 3 weeks and combined with trastuzumab (initial dose of $8 \mathrm{mg} / \mathrm{kg}$ intravenously (iv) day 1 , and $6 \mathrm{mg} / \mathrm{kg}$ iv every 3 weeks) prior to surgery. This treatment induced pCR in $41.4 \%$ of patients, and breast conservation was possible in the majority of patients. These results supported the initiation of the largest clinical trial on neoadjuvant therapy of HER2-positive breast cancer, the GeparQuattro study [26]. The multicenter study conducted by the AGO and German Breast Group (GBG) evaluated capecitabine, as added to a standard therapy, and the benefit of trastuzumab in the neoadjuvant treatment. Overall, 1,509 patients started chemotherapy with 4 cycles of EC. Subsequently, 1,421 patients were randomized to be treated with docetaxel only $(\mathrm{T}, \mathrm{n}=471)$, docetaxel/ capecitabine (TX, $\mathrm{n}=471$ ), or sequential treatment of docetaxel followed by capecitabine $(T-X, n=479)$, or, in patients with HER2-positive tumors, additional trastuzumab. The percentage of pCR was $22.3 \%$ (T), $19.5 \%$ (TX), and $22.3 \%$ (T-X). Breast conservation was possible in $70.1,68$, and $65.3 \%$ of patients. The final analysis concluded that adding capecitabine to or prolonging the duration of neoadjuvant EC plus docetaxel does not result in higher efficacy at surgery but might impair the safety profile of this regimen [27].

The data from the GeparQuattro study have been furthermore evaluated separately for HER2-positive and -negative tumors [28]. Out of 1,509 participants, 445 had HER2-positive tumors treated with trastuzumab and chemotherapy. The pCR rate was $31.7 \%$ which was $16 \%$ higher than that in the reference group $(15.7 \%)$. HER2-positive patients without response to the first 4 cycles of EC showed a high pCR rate of 


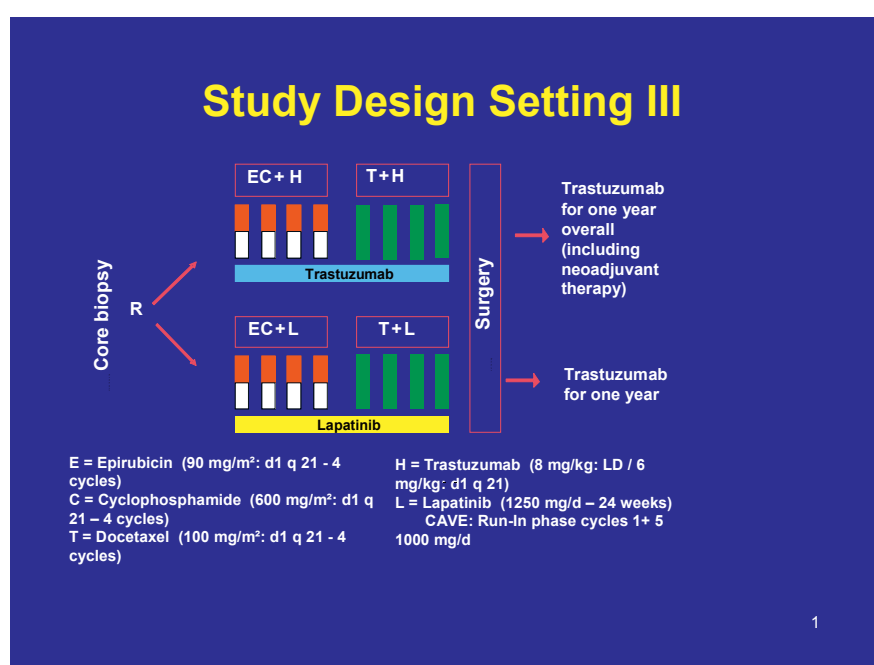

Fig. 2. Design of the Geparquinto study.

$16.6 \%(3.3 \%$ in the reference group). Breast conservation rate was $63.1 \%$ and comparable to the reference group (64.7\%). This study confirms that the neoadjuvant combination of trastuzumab and an anthracycline-taxane-based chemotherapy results in a higher rate of pCR with no clinically relevant increase in toxicity. The superiority of neoadjuvant trastuzumab in HER2-positive disease could also be verified in the international NeOAdjuvant-Herceptin (NOAH) study including 327 patients with locally advanced breast cancer [29]. Prior to surgery, patients were treated with a chemotherapy containing anthracyclines and taxanes with or without trastuzumab. Additional trastuzumab could nearly double the pCR from $23 \%$ in the control group to $43 \%$ in the trastuzumab group. The final analysis, performed after a median follow-up of 3 years, showed a correlation of the high pCR rate and a significant improvement of event-free survival (53 vs. $70 \% ; \mathrm{p}=0.0006$ ) [30]. This promising approach is supported by data from the MD Anderson Cancer Center with a pCR rate of more than $60 \%$ when trastuzumab is given concomitantly with an anthracycline-containing chemotherapy [31].

Patients included in the Neo-ALTTO study were treated with lapatinib or trastuzumab or a combination of both prior to surgery. The regimen started with this medication for 6 weeks, and was then combined with chemotherapy with paclitaxel $80 \mathrm{mg} / \mathrm{m}^{2}$ weekly for 12 weeks, followed by 4 cycles EC $90 / 600 \mathrm{mg} / \mathrm{m}^{2}$. Endpoint was pCR as compared between any of the lapatinib groups and the trastuzumab group. In the GeparQuinto study, trastuzumab and lapatinib are compared in combination each with an anthracycline- or taxane-containing chemotherapy (fig. 2, study design GeparQuinto). First results, which have been presented at the San Antonio Breast Cancer Symposium 2009, showed that lapatinib is well tolerated in this setting and dosage, and that the therapy can be performed safely [32]. In the Cher Lob study [33], the dose of lapatinib was reduced to $1,250 \mathrm{mg} / \mathrm{d}$ when combined with pa- clitaxel weekly, and to $750 \mathrm{mg} / \mathrm{d}$ when added to a combination of paclitaxel and trastuzumab, because the frequency of diarrhea was too high. The GeparQuintto program is also exploring the integration of bevacizumab and everolimus (RAD001) into current neoadjuvant chemotherapy regimes for primary breast cancer. Furthermore, the open, multicenter, one-armed phase II study SOFIA has been initiated to evaluate efficacy and tolerability of sorafenib, a multikinase inhibitor, in the neoadjuvant treatment of women suffering from primary breast cancer.

Further novel, targeted agents such as lapatinib, bevacizumab, T-DM1, pertuzumab, and neratinib are currently evaluated in clinical trials. Pertuzumab, a recombinant humanized monoclonal antibody (2C4), binds to extracellular domain II of the HER2 receptor and blocks its ability to dimerize with other HER family receptors (HER1, HER2, HER3, HER4). Pertuzumab is the first in a new class of targeted agents known as HER dimerization inhibitors (HDIs). The drug showed promising activity with trastuzumab in the treatment of metastatic breast cancer in a phase II study [34]. Phase III trials will explore the effectiveness of pertuzumab in combination with trastuzumab as first-line therapy in metastatic disease (CLEOPATRA study) as well as in the neoadjuvant setting in early breast cancer. T-DM1 consists of the trastuzumab monoclonal antibody, chemically fused to a cytotoxin - a potent microtubule inhibitor called maytansine that is derived from a sea sponge. The drug appeared to improve outcomes in heavily pretreated metastatic breast cancer [35]. Median progression-free survival was 7.3 months with single agent trastuzumab-DM1 (T-DM1) in a phase II study. The objective response rate was $32.7 \%$ in women whose tumors had progressed after treatment with an anthracycline, taxane, capecitabine, trastuzumab, and lapatinib, including two HER2-targeted agents in the metastatic setting. Moreover, the investigational agent was well tolerated, with no dose-limiting cardiotoxicity. The drug is currently developed in phase III studies for first-line treatment. Neratinib is an orally administered irreversible inhibitor of the HER2 and EGFR kinases. The phase II study FB-7 explores neratinib in combination with weekly paclitaxel followed by AC as neoadjuvant therapy for women with HER2-positive, locally advanced breast cancer. Patients in the control arm will receive neoadjuvant trastuzumab in combination with weekly paclitaxel followed by AC. The primary aim of the study is to determine the pCR rate in breast and axillary nodes following the neoadjuvant therapy regimens.

\section{Future Aspects}

Adding trastuzumab to chemotherapy has dramatically improved the prognosis of patients with HER2-positive breast cancer. Further progress is ongoing by continuously improving schedules and combinations. With the increasing under- 
standing of the molecular biology of breast cancer, several other important intracellular pathways have been identified as targets for novel therapeutic agents, including other members of the HER family, angiogenic pathways, proliferative pathways, pathways of cell cycle regulation, apoptosis pathways, and many others. It is also becoming clearer that the complex interaction of these pathways will likely require multiple targets to be inhibited to optimize cytotoxicity and over- come resistance mechanisms. This review highlights targeted agents for early and locally advanced breast cancer.

\section{Conflict of Interest}

The author does not have a conflict of interests. The author is a member of advisory boards of Roche and GSK, all honoraria are paid to HELIOS Klinikum Berlin-Buch.

\section{References}

1 Beatson G: On the treatment of carcinoma of the mamma: suggestions for a new method of treatment, with illustrative cases. Lancet 1896;2:104107

2 Buzdar AU: Advances in endocrine treatments for postmenopausal women with metastatic and early breast cancer. Oncologist 2003;8:335-341.

3 Slamon DJ, Leyland-Jones B, Shak S, et al.: Use of chemotherapy plus a monoclonal antibody against HER2 for metastatic breast cancer that overexpresses HER2. N Engl J Med 2001;344:783-792.

4 Extra JM, Cognetti F, Chan S, et al.: First-line trastuzumab (Herceptin) plus docetaxel versus docetaxel alone in women with HER2-positive metastatic breast cancer (MBC): results from a randomised phase II trial (M77001). Breast Cancer Res Treat 2003;82(suppl 1):S47(abstr 217).

5 Joensuu H, Kellokumpu-Lehtinen PL, Bono P, et al.; FinHer Study Investigators: Adjuvant docetaxel or vinorelbine with or without trastuzumab for breast cancer. N Engl J Med 2006;354:809-820.

6 Perez E, Romond EH, Suman VJ, et al.: Updated results of the combined analysis of NCCTG N9831 and NSABP B-31 adjuvant chemotherapy with/ without trastuzumab in patients with HER2-positive breast cancer. Proc ASCO 2007, J Clin Oncol 25 (suppl 1):512.

7 Slamon DJ, Eiermann W, Robert N, et al.: Phase III randomized trial comparing doxorubicin and cyclophosphamide followed by docetaxel $(\mathrm{AC} \rightarrow \mathrm{T})$ with doxorubicin and cyclophosphamide followed by docetaxel and trastuzumab ( $\mathrm{AC} \rightarrow \mathrm{TH})$ with docetaxel, carboplatin and trastuzumab $(\mathrm{TCH})$ in HER2-positive early breast cancer patients: BCIRG 006 study. Breast Cancer Res Treat 2005; 94(suppl 1):S5.

$\checkmark 8$ Smith I, Procter M, Gelber RD, et al.: HERA study team. 2-year follow-up of trastuzumab after adjuvant chemotherapy in HER2-positive breast cancer: a randomised controlled trial. Lancet 2007; 369:29-36.

9 Viani GA, Afonso SL, Stefano EJ, et al.: Adjuvant trastuzumab in the treatment of her-2-positive early breast cancer: a meta-analysis of published randomized trials. BMC Cancer 2007;7:153.

10 Joensuu H, Bono P, et al.: Update of the FINHER trial based on 5 years of follow-up. Breast 2009; 18(suppl 1):abstr S24.

11 National Cancer Institute: Trastuzumab for 6 months or 1 year in treating women with nonmetastatic breast cancer that can be removed by surgery. clinicaltrials.gov/show/NCT00381901.
12 Guarneri V, Frassoldati A, Bruzzi P, et al.: Multicentric, randomised phase III trial of two different adjuvant chemotherapy regimens plus three versus twelve months of trastuzumab in patients with HER2-posotive breast cancer (Short-HER Trial; NCT00629278) Clin Breast Cancer 2008;8:453-456.

13 Gianni L, Goldhirsch A, Gelber RD, et al.: Update of the HERA trial and the role of 1 year trastuzumab as adjuvant therapy for breast cancer. Breast 2009;18(suppl 1):abstr S25.

14 Untch M, Gelber RD, Jackisch C, et al.; HERA Study Team: Estimating the magnitude of trastuzumab effects within patient subgroups in the HERA trial. Ann Oncol 2008;18:1090-1096.

15 Rakkhit R, Broglio K, Peintinger F, et al.: Significant increased recurrence rates among breast cancer patients with HER2-positive, T1a,b N0M0 tumors. San Antonio Breast Cancer Symposium 2008; abstr 701.

16 Joensuu H, Isola J, Lundin M, et al.: Amplification of erbB2 and erbB2 expression are superior to estrogen receptor status as risk factors for distant recurrence in pT1N0M0 breast cancer: a nationwide population-based study. Clin Cancer Res 2003;9: 923-930.

17 Chia S, Norris B, Speers C, et al.: Human epidermal growth factor receptor 2 overexpression as a prognostic factor in a large tissue. J Clin Oncol 2008;26: 5697-5704.

18 Curigliano G, Viale G, Bagnardi V, et al.: Clinical relevance of HER2 overexpression/amplification in patients with small tumor size and node-negative breast cancer. J Clin Oncol 2009;27:5693-5699.

19 Gonzalez-Angulo AM, Litton JK, Broglio KR, et al.: High risk of recurrence for patients with breast cancer who have human epidermal growth factor receptor 2-positive, node-negative tumors $1 \mathrm{~cm}$ or smaller. J Clin Oncol 2009;27:5700-5706.

20 Slamon DJ, Eiermann W, Robert N, et al.: BCIRG 006: 2nd interim analysis phase III randomized trial comparing doxorubicin and cyclophosphamide followed by docetaxel $(\mathrm{AC} \rightarrow \mathrm{T})$ with doxorubicin and cyclophosphamide followed by docetaxel and trtastuzumab $(\mathrm{AC} \rightarrow \mathrm{TH})$ with docetaxel, carboplatin and trastuzumab (TCH) in Her2neu positive early breast cancer patients. San Antonio Breast Cancer Symposium 2006; abstr 52.

21 Slamon DJ, Eiermann W, Robert N, et al.: BCIRG 006: Phase III randomized trial comparing doxorubicin and cyclophosphamide followed by docetaxel $(\mathrm{AC} \rightarrow \mathrm{T})$ with doxorubicin and cyclophosphamide followed by docetaxel and trastuzumab $(\mathrm{AC} \rightarrow \mathrm{TH})$ with docetaxel, carboplatin and trastuzumab (TCH) in Her2neu positive early breast cancer patients: BCIRG 006 Study. San Antonio Breast Cancer Symposium 2009; abstr 62.
22 Geyer CE, Forster J, Lindquist D, et al.: Lapatinib plus capecitabine for HER2-positive advanced breast cancer. N Engl J Med 2006;355:2733-2743.

23 Wolmark N, Wang J, Mamounas E, et al.: Preoperative chemotherapy in patients with operable breast cancer: nine-year results from national surgical adjuvant breast and bowel project B-18. J Natl Cancer Inst Monogr 2003;30:96-102.

24 Fisher B, Brown A, Mamounas E, Wieand S, Robidoux A, Margolese RG, et al.: Effect of preoperative chemotherapy on local-regional disease in women with operable breast cancer: findings from National Surgical Adjuvant Breast and Bowel Project B-18. J Clin Oncol 1997;15:2483-2489.

25 Untch M, Stoeckl D, Konecny G, et al.: A multicenter phase II study of preoperative epirubicin, cyclophosphamide (EC) followed by paclitaxel (P) plus trastuzumab $(\mathrm{T})$ in Her2 positive primary breast cancer. Breast Cancer Res Treat 2005; 94(suppl 1):abstr 1064.

26 Untch M, Rezai M, Loibl S, et al.: Neoadjuvant treatment of HER2 overexpressing primary breas cancer with trastuzumab given concomitantly to epirubicin/cyclophosphamide followed by docetaxel and capecitabine. First analysis of efficacy and safety of the GBG/AGO multicenter intergroup-study 'GeparQuattro'. Eur J Cancer 2008;suppl 6:abstr 47.

27 Von Minckwitz G, Rezai M, Loibl S, et al.: Capecitabine in addition to anthracycline/taxane-based neoadjuvant treatment in patients with primary breast cancer: the phase III GeparQuattro study. J Clin Oncol 2010;28:2015-2023.

28 Untch M, Rezai M, Loibl S, et al.: Neoadjuvant treatment with trastuzumab in HER2-positive breast cancer: results from the GeparQuattro study. J Clin Oncol 2010;28:2024-2031.

29 Buzdar AU, Hunt K, Smith T, et al.: Significantly higher pathological complete remission (pCR) rate following neoadjuvant therapy with trastuzumab $(\mathrm{H})$, paclitaxel $(\mathrm{P})$ and anthracycline containing chemotherapy $(\mathrm{CT})$ : Initial results of a randomized trial in operable breast cancer (BC) with HER/2 positive disease. Proc AM Soc Clin Oncol 2004; abstr 520.

30 Gianni L, Eiermann W, Semiglazov V, et al.: Neoadjuvant trastuzumab in patients with HER2positive locally advanced breast cancer: primary efficacy analysis of the NOAH trial. Cancer Res 2009;69(suppl 2):abstr 31.

31 Buzdar AU, Valero VV, Ibrahim NK, et al.: Neoadjuvant therapy with paclitaxel followed by 5-fluorouracil, epirubicin, and cyclophosphamide chemotherapy and concurrent trastuzumab in human epidermal growth factor receptor 2-positive operable breast cancer: an update of the initial randomized study population and data of additional patients treated with the same regimen. Clin Cancer Res 2007;13:228-233. 
32 Untch M, Kaufmann M, Hilfrich J: Lapatinib can safely be given concomitant to EC-T as neoadjuvant chemotherapy for breast cancer. First planned safety analysis of the Geparquinto study (GBG 44). San Antonio Breast Cancer Symposium 2009; abstr 1094 .

33 Guarneri V, Frassoldati A, Piacentini F, et al.: Preoperative chemotherapy plus lapatinib or trastuzumab or both in HER2-positive operable breast cancer (CHERLOB Trial). Clin Breast Cancer 2008; 8:192-194.

34 Baselga J, Cameron D, et al.: Objective response rate in a phase II multicenter trial of pertuzumab (P), a HER2 dimerization inhibiting monoclonal antibody, in combination with trastuzumab (T) in patients (Pts) with HER2 positive metastatic breast cancer (MBC) which had progressed during trastuzumab therapy. Proc Ann Meet Am Soc Clin Oncol 2007; abstr 1004.

35 Krop I, LoRusso P, et al.: A phase II study of trastuzumab-DM1 (T-DM1), a novel HER2 antibodydrug conjugate, in patients with HER2+ metastatic breast cancer who were previously treated with an anthracycline, a taxane, capecitabine, lapatinib, and trastuzumab. SABCS 2009; abstr 710 .

36 Park Y, Kim S, Ok O, et al.: Risk stratification by hormonal receptor (ER, PgR) and HER2 status in small $(\leq 1 \mathrm{~cm})$ invasive breast cancer: Who might be a possible candidate for adjuvant treatment? J Clin Oncol 2009;15s(suppl):abstr 564.

37 Smith I, Procter M, Gelber RD, et al.: 2-year follow-up of trastuzumab after adjuvant chemotherapy in HER2-positive breast cancer: a randomised controlled trial. Lancet 2007;369:29-36.

38 Spielmann M, Roche H, Humblet Y, et al.: 3-year follow-up of trastuzumab fallowing adjuvant chemotherapy in node positive HER2-positive breast cancer patients: results of the PACS-04 trial. Breast Cancer Res Treat 2007;106 (suppl 1):S19, abstr 72.

39 Amar S, Ann ME, Geiger XJ, et al.: Prognosis and clinical outcome of patients with node negative $<$ or $=1 \mathrm{~cm}$ breast cancer. Breast Cancer Res Treat 2007;106 (suppl 1):-S254, abstr 6024.

40 Tovey SM, Marla S, Brown S, et al.: Poor survival outcomes in HER2 positive breast cancer patients with low grade, node negative tumours. Implications for trastuzumab therapy? SABCS 2008:abstr 702.

41 Pagani O, Gelber S, Simoncini E, et al.: Is adjuvant chemotherapy of benefit for postmenopausa women who receive endocrine treatment for highly endocrine-responsive, node-positive breast cancer? International Breast Cancer Study Group Trials VII and 12-93. Breast Cancer Res Treat 2009;116 (3):491-500.

42 Norris B, Chia S, Cheang M, et al.: Poor $10 \mathrm{yr}$ breast cancer specific survival (BCSS) and relapse free survival (RFS) for HER-2 positive T1pN0 tumors. Breast Cancer Res Treat 2006;100 (suppl 1): S90, abstr 2031. 